\title{
Intense Ion Beam Transport in Magnetic Quadrupoles: Experiments on Electron and Gas Effects
}

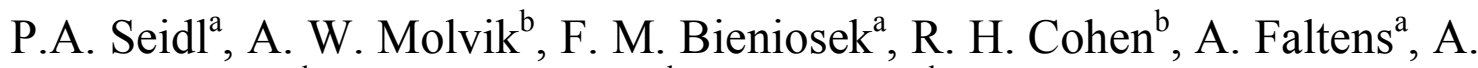

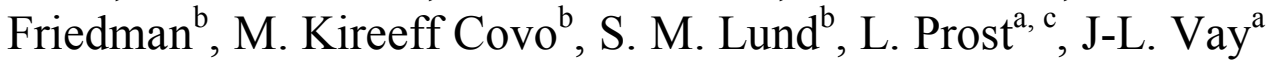 \\ ${ }^{a}$ Lawrence Berkeley National Laboratory, Berkeley CA 94720, USA \\ ${ }^{b}$ Lawrence Livermore National Laboratory, Livermore, CA 94550, USA. \\ ${ }^{c}$ Present address: Fermi National Accelerator Laboratory, Batavia, IL 60510, USA
}

\begin{abstract}
Heavy-ion induction linacs for inertial fusion energy and high-energy density physics have an economic incentive to minimize the clearance between the beam edge and the aperture wall. This increases the risk from electron clouds and gas desorbed from walls. We have measured electron and gas emission from $1 \mathrm{MeV} \mathrm{K}^{+}$incident on surfaces near grazing incidence on the High-Current Experiment (HCX) at LBNL. Electron emission coefficients reach values $>100$, whereas gas desorption coefficients are near $10^{4}$. Mitigation techniques are being studied: A bead-blasted rough surface reduces electron emission by a factor of 10 and gas desorption by a factor of 2 . We also discuss the results of beam transport (of 0.03-0.18 $\mathrm{A} \mathrm{K}^{+}$) through four pulsed room-temperature magnetic quadrupoles in the HCX at LBNL. Diagnostics are installed on HCX, between and within quadrupole magnets, to measure the beam halo loss, net charge and expelled ions, from which we infer gas density, electron trapping, and the effects of mitigation techniques. A coordinated theory and computational effort has made significant progress towards a self-consistent model of positiveion beam and electron dynamics. We are beginning to compare experimental and theoretical results.
\end{abstract}

\section{INTRODUCTION}

Electron cloud effects [1] and beam-induced pressure rises [2], which are frequently observed to limit the beam quality in high-energy physics colliders and high-intensity rings, are also a concern for future high-intensity heavy ion linear accelerators such as envisioned in Heavy Ion Inertial Fusion (HIF) [3]. The High Current Experiment (HCX) at Lawrence Berkeley National Laboratory is part of the US program to explore heavy-ion beam transport at a scale representative of the low-energy end of an induction linac driver for fusion energy production. The primary mission of this experiment is to investigate aperturefilling factors acceptable for the transport of spacecharge-dominated heavy-ion beams at high spacecharge intensity (line charge density up to $\sim 0.2$ $\mu \mathrm{C} / \mathrm{m})$ over long pulse durations $(>4 \mu \mathrm{s})$ in alternating-gradient electrostatic and magnetic quadrupoles. This experiment is testing -- at driver relevant scale -- transport issues resulting from nonlinear space-charge effects and collective modes, beam centroid alignment and beam steering, matching, image charges, halo, electron cloud effects, and longitudinal bunch control.

At an injection energy of 1-1.8 MeV, a line-charge density of $0.1<\lambda<0.2 \mu \mathrm{C} \mathrm{m}^{-1}$ and a 5 - $\mu$ S FWHM pulse duration, the HCX main beam parameters are in the range of interest for a fusion driver front-end. At 1 $\mathrm{MeV}$ where we performed our experiments, the generalized beam perveance is

$$
K=\frac{2 q I}{4 \pi \varepsilon_{0} m(\beta \gamma c)^{3}}=8 \times 10^{-4} .
$$

The number of $\mathrm{K}^{+}$ions per bunch is $6 \times 10^{12}$. While the driver front-end would have $\sim 10^{14}$ particles/bunch distributed over $\sim 20 \mu \mathrm{s}$.

In this regime space-charge forces strongly influence the beam properties, and the image charges induced on metallic structures of the machine aperture play an important role. 
This paper focuses on intense beam transport in magnetic quadrupoles, where electrons and gas effects may perturb beam quality. The beam potential (1-2 $\mathrm{kV}$ ) may trap generated electrons, depending on their initial conditions and thereby change the space-charge potential.

\section{EXPERIMENTS AND DIAGNOSTICS}

The 2-MV injector, matching section and electrostatic transport section precede the apparatus depicted in Fig. 1, and deliver a $0.18 \mathrm{~A}$, low-emittance $\left(\varepsilon_{\mathrm{n}}=4 \beta \gamma \varepsilon_{\mathrm{rms}}=0.5 \pi \mathrm{mm}\right.$-mrad $) \mathrm{K}^{+}$beam that is diagnosed at the $\mathrm{D} 2$ diagnostic station with double slit scanners and alumina-scintillator optical diagnostics. In previous experiments, no emittance growth and $<1 \%$ beam loss was observed through the preceding 10 quadrupole electrostatic transport section for cases in which the $2 \cdot r m s$ beam envelope filled 60 and $80 \%$ of the physical aperture $\left(\mathrm{R}_{\mathrm{ap}}=23 \mathrm{~mm}\right)$. The results were for an ambient pressure of $\approx 2 \times 10^{-7}$ Torr. Electrons produced by beam-background gas interactions or from halo loss at limiting apertures are effectively cleared by the fields established by the quadrupole electrodes at $\pm 17-25 \mathrm{kV}$. These electrostatic transport results [4] are an electron-free comparison to transport in magnetic quadrupoles. While electrostatic quadrupoles are suitable at low energy, magnetic quadrupoles are desireable for most of a HIF driver.

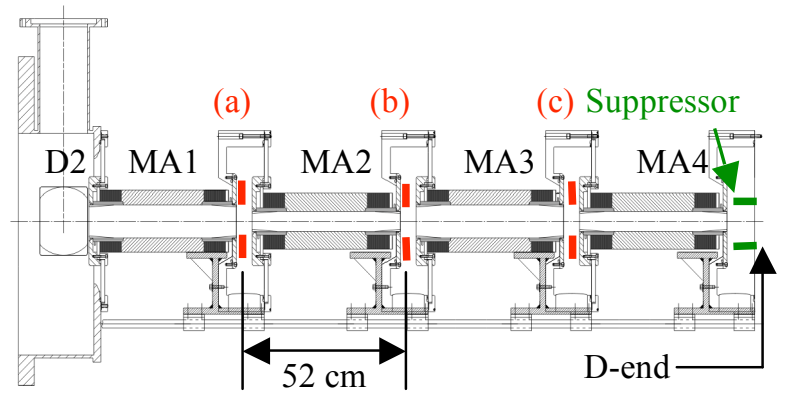

FIGURE 1. An elevation view of the magnetic quadrupole lattice in the HCX. Clearing electrodes are marked in red, and the $\mathrm{e}^{-}$suppressor is green.

The Gas-Electron Source Diagnostic installed at the end of the HCX beamline was used to measure $\mathrm{e}^{-}-$ emission and gas-desorption coefficients $\eta_{\mathrm{e}}$ and $\eta_{\mathrm{o}}$ respectively, due to ion bombardment of metal surfaces near grazing incidence [5]. The measured yields will enable us to interpret electron emission currents from electrodes in beam tubes due to beamhalo loss, and the resulting gas desorption. $\eta_{\mathrm{e}}(\theta)$, which reaches 130 at $88^{\circ}$, is observed to scale as $\eta_{\mathrm{e}} \propto 1 / \cos (\theta),(\theta=0$ is normal incidence $)$, similar to the angular dependence at higher ion energies [6]. $\eta_{\mathrm{o}}(\theta)$, measured from the pressure rise after a pulse, is less steep than $1 / \cos (\theta)$, varying from $6 \times 10^{3}$ to $9 \times 10^{3}$ for $80 \leq \theta \leq 88^{\circ}$.

Roughening the steel surface (glass bead blasting) reduced the fraction of the area on which an ion can impinge at grazing incidence, resulting in most ions striking at angles closer to normal incidence. We found that $\eta_{\mathrm{e}}$ and $\eta_{0}$ were independent of the macroscopic target angle. Furthermore, in the range $80^{\circ} \leq \theta \leq 88^{\circ}, \eta_{\mathrm{e}}$ decreased by a factor $3-10$ compared to the smooth surface, and $\eta_{0}$ decreased by a factor 1.7-2.4. This reduction in yield represents a promising mitigation technique for low-energy ion beamlines, where the ions have a short $(\sim 1 \mu \mathrm{m})$ range in metal.

The magnetic quadrupole section has a lattice period of $104 \mathrm{~cm}$. The pulsed $(\Delta \mathrm{t}=0.8 \mathrm{~ms})$ magnetic quadrupoles have a 6 x $10 \mathrm{~cm}$ elliptical aperture, magnetic length of $31 \mathrm{~cm}$, and are operated at $\approx 10$ $\mathrm{T} / \mathrm{m}$. They were designed as a prototype for a higher energy, multiple beam array [7].

Cylindrical clearing electrodes $(\mathrm{V}=+9 \mathrm{kV})$ between each pair of magnets collect electrons that accumulate in the beam path, and collect electrons that would otherwise stream from one magnet to another. A negatively biased $\mathrm{e}^{-}$suppressor $(\mathrm{V}=-10 \mathrm{kV})$ between the last magnet (MA4) and the diagnostic end station (D-end) effectively prevents electrons produced at intercepting beam diagnostics (D-end) from flowing upstream through the magnetic lattice.

Diagnostics are mounted on octagonal tubes that fit the elliptical beam tube bore in magnets MA3 and MA4, with $\approx 0.75 \mathrm{~cm}$ clearance between the octagonal diagnostics mounting tube and the elliptical magnet bore for the recessed diagnostics and cables. These diagnostics include capacitive electrodes to measure the net beam charge, electrodes shielded from the beam electric field by grids to measure the current of expelled ions from gas ionization in MA4, and flush electrodes running the length of the octagonal tube in MA3 (two per quadrant) [8].

For an apertured beam $(\mathrm{I}=0.03 \mathrm{~A})$ the envelope parameters are understood with PIC simulations of ion transport without electron effects and fills $\approx 50 \%$ of the magnet aperture. The 0.18 -A (un-apertured) beam fills $\approx 70 \%$ of the aperture. With clearing electrodes and electron suppressor biased, the magnet diagnostics show larger signals and the downstream beam distribution shows signs of distortion resembling simulated electron effects. 


\section{THEORETICAL MODELING}

A new computational method for the selfconsistent tracking of electrons and ions has been developed, and has been added to the WARP 3D particle in cell code. It is an $\mathrm{e}^{-}$-tracking algorithm that interpolates between full electron dynamics and drift kinetics. This enables the use of a time-step of the same order as the $\mathrm{e}^{-}$cyclotron period within the magnets $\left(\approx 10^{-10} \mathrm{sec}\right)$. Modules for secondary $\mathrm{e}^{-}$ emission and ion-induced electron emission have also been added to the simulations [9].

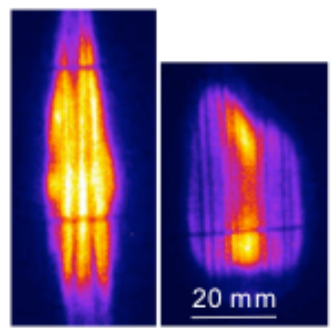

FIGURE 2. $J(x, y)$ as measured with a scintillator at D-end. Left: electron suppressor grounded. Right: electron suppressor $\mathrm{V}=-10 \mathrm{kV}$.

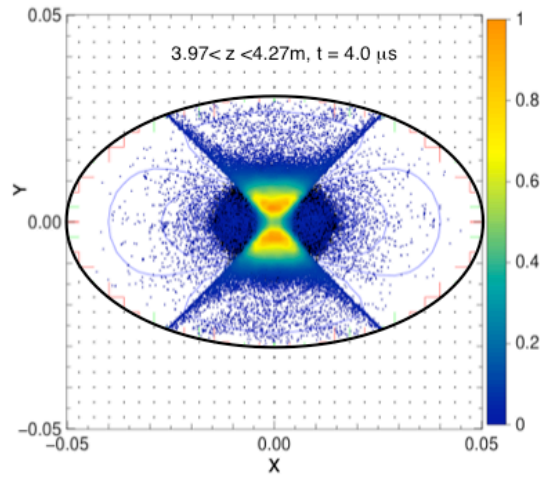

FIGURE 3. Simulation of the experiment with the electron source from the intercepting diagnostic plate after MA4. The $\mathrm{K}^{+}$ions are in a roughly elliptical distribution (black), and the $\mathrm{e}^{-}$density is color coded according to the scale on the right. Electrostatic potentials are in blue.

By comparing the transverse beam distributions (for the suppressor grounded) to the model, we are beginning to validate PIC models of electron accumulation in the magnetic quadrupole lattice. The experimental $\mathrm{J}(\mathrm{x}, \mathrm{y})$ distribution shows dramatic differences depending on the suppressor bias (Fig. 2). The beam is more vertically elongated when the suppressor is grounded because electrons desorbed on the diagnostic plate downstream of the suppressor back-stream and partially neutralize the beam in the drift region upstream and in the quadrupole bores.
The $\mathrm{e}^{-}$dynamics in the magnetic lattice are complicated, depending on the beam potential, applied magnetic field, and clearing electrode bias. Figure 3 shows that the simulated electron distribution in MA4 occupies mainly the top and bottom quadrants of the magnetic field geometry. The simulations also show that the electron population decreases steeply in each successive upstream quadrupole.

\section{ACKNOWLEDGEMENTS}

We are grateful to R. Hipple, C. Rogers and their staff for excellent technical support. This work performed under the auspices of the U.S Department of Energy by University of California, Lawrence Livermore and Lawrence Berkeley National Laboratories under contracts No. W-7405-Eng-48 and DE-AC03-76SF00098.

\section{REFERENCES}

1 Proc. ECLOUD'02 Workshop, CERN, 15-18 April 2002 CERN-2002-001 http://wwwslap.cern.ch/collective/ ecloud02/proceedings/index.html

2. W. Y. Zhang, in ICFA Workshop on Beam Induced Pressure Rise in Rings, http://www.c-ad.bnl.gov/icfa (2003).

3. R. O. Bangerter, Phil. Trans. R. Soc. Lond. A 357, 575 (1999).

4. L. Prost, et al., " High current transport experiment for heavy ion inertial fusion", to be published in PRST-AB.

5. A. W. Molvik, et al., Phys. Rev. ST-AB 7, 093202 (2004).

6. P. Thieberger, A. L. Hanson, D. B. Steski, S. Y.Z. V. Zajic, and H.Ludewig, Phys. Rev. A. 61, 042901 (2000).

7. A. Faltens et al., in Proc. Part. Accel. Conf. 1999, http://accelconf.web.cern.ch/accelconf/p99/PAPERS/TH P159.PDF

8. A.W. Molvik, et al., "Experimental Studies of Electrons in a Heavy-Ion Beam", to be published in Proc. HIF-04, $\mathrm{NIM}-\mathbf{A}$.

9. R.H. Cohen, et al., "Simulating Electron Cloud Effects In Heavy Ion Accelerators", to be published in Proc. HIF 04, NIM - A. and R.H. Cohen et al., "Comparison of Electron Cloud Simulation and Experiments in the HighCurrent Experiment" to be published in Proc. $46^{\text {th }}$ Annual Meeting of the Division of Plasma Physics, Savannah, USA Nov 15-19, 2004. 